\title{
Chronic Kidney Disease: Novel Insights from Genome-Wide Association Studies
}

\author{
Carsten A. Böger ${ }^{\text {a }} \quad$ Iris M. Heid ${ }^{b, c}$ \\ Departments of ${ }^{\mathrm{a}}$ Internal Medicine II and ${ }^{\mathrm{b}}$ Epidemiology and Preventive Medicine, University Hospital \\ Regensburg, Regensburg, and ' Institute of Epidemiology, Helmholtz Zentrum München, German Research Center \\ for Environmental Health, Neuherberg, Germany
}

\section{Key Words}

Genome-wide association study • Chronic kidney disease •

End-stage renal disease - Diabetic nephropathy •

Membranous nephropathy $\cdot \lg A$ nephropathy

Albuminuria $\cdot$ Glomerular filtration rate $\cdot$ Progression of

chronic kidney disease $\cdot$ Kidney function decline

\begin{abstract}
Chronic kidney disease (CKD) is common, affecting about $10 \%$ of the general population, and causing significant morbidity and mortality. Apart from the risk conferred by traditional cardiovascular risk factors, there is a strong genetic component. The method of a genome-wide association study (GWAS) is a powerful hypothesis-free approach to unravel this component by association analyses of CKD with several million genetic variants distributed across the genome. Since the publication of the first GWAS in 2005, this method has led to the discovery of novel loci for numerous human common diseases and phenotypes. Here, we review the recent successes of meta-analyses of GWAS on renal phenotypes. UMOD, SHROOM3, STC1, LASS2, GCKR, ALMS1, TFDP2, DAB2, SLC34A1, VEGFA, PRKAG2, PIP5K1B, ATXN2/SH2B3, DACH1, UBE2Q2, and SLC7A9 were uncovered as loci associated with estimated glomerular filtration rate (eGFR) and CKD, and CUBN as a locus for albuminuria in cross-sectional data of general population studies. However, less than $1.5 \%$ of the
\end{abstract}

total variance of eGFR and albuminuria is explained by the identified variants, and the relative risk for CKD is modified by at most $20 \%$ per locus. In African Americans, much of the risk for end-stage nondiabetic kidney disease is explained by common variants in the MYH9/APOL1 locus, and in individuals of European descent, variants in HLA-DQA1 and $P L A_{2} R 1$ implicate most of the risk for idiopathic membranous nephropathy. In contrast, genetic findings in the analysis of diabetic nephropathy are inconsistent. Uncovering variants explaining more of the genetically determined variability of kidney function is hampered by the multifactorial nature of CKD and different mechanisms involved in progressive CKD stages, and by the challenges in elucidating the role of lowfrequency variants. Meta-analyses with larger sample sizes and analyses of longitudinal renal phenotypes using higherresolution genotyping data are required to uncover novel loci associated with severe renal phenotypes.

Copyright $\odot 2011$ S. Karger AG, Basel

\section{Rationale for Genetic Studies in Kidney Disease}

Chronic kidney disease (CKD) affects about $10 \%$ of the general population in industrialized nations, incurring high morbidity and mortality, and posing a significant financial burden to the health care systems [1-3], with patients that progress to end-stage renal disease

\section{KARGER}

Fax +4161306 1234

E-Mail karger@karger.ch

www.karger.com
(C) 2011 S. Karger AG, Basel

$1420-4096 / 11 / 0344-0225 \$ 38.00 / 0$

Accessible online at:

www.karger.com/kbr
PD Dr. Carsten A. Böger

Department of Internal Medicine II, Nephrology

University Hospital Regensburg

Franz-Josef-Strauss-Allee 11, DE-93053 Regensburg (Germany)

Tel. +49941944 7301, E-Mail carsten.boeger@ klinik.uni-regensburg.de 
(ESRD) particularly affected. Diabetes and hypertension are major risk factors, but do not account for all of the risk [4].

Heritability of glomerular filtration rate (GFR) has been estimated to range from 36 to $75 \%$, and from 16 to $49 \%$ for albuminuria, one of the first signs of kidney damage $[5,6]$. This, and the multitude of monogenic kidney diseases involving a particularly severe phenotype with onset in early childhood, e.g. congenital nephrotic syndrome of the Finnish type (NPHS1), or in adulthood, e.g. autosomal dominant polycystic kidney disease [7-11], are the rationale for searching for common variants associated with renal function and kidney disease phenotypes in the general population [5,11-13]. The elucidation of the genetic variants involved may lead to a better understanding not only of variability of GFR and albuminuria in the general population but also of the biology of clinical phenotypes such as CKD, progressive kidney function decline and ESRD. Ultimately, this could lead to novel tools for diagnosis, prevention and therapy of CKD $[5,12,13]$.

\section{The Evolving Methodology in Genetic Research}

Classical genetic mapping approaches (linkage analyses) in families with index patients affected by a rare disease have long been and continue to be successful in discovering mutations causing rare single-gene diseases with a clear mendelian mode of inheritance and mostly a pathognomonic clinical phenotype [7]. However, the use of these hypothesis-free methods has not been overly successful in identifying genetic variants associated with common diseases (ESRD and diabetic nephropathy) or quantitative kidney traits (GFR and albuminuria). While carnosinase (CNDP1) was identified as a likely candidate gene for diabetic nephropathy [14-19], other studies on diabetic nephropathy or other kidney phenotypes have not been consistent [20-33].

Another approach investigated the association between common genetic variants (minor allele frequencies $>5 \%$ in the general population) in plausible candidate genes and kidney function or disease phenotypes such as diabetic nephropathy $[11,34]$. However, confirmatory replication was rarely achieved due to a multitude of study design issues such as inadequate power, low significance threshold, and differences in phenotype definition between studies [35].

A further hypothesis-free approach is provided by genome-wide association studies (GWAS). The GWAS approach has been catalyzed by the publication of the human genome just over 10 years ago [36, 37], by technological advances in the high-throughput detection of genome sequence variation and the unraveling of the architecture of genetic variation in humans of different ethnic origin in the HAPMAP project [38]. With the highthroughput genotyping technologies offered by companies such as Illumina and Affymetrix, it is now possible to rapidly genotype more than 1 million single nucleotide polymorphisms (SNPs) across the whole genome per person in a single analytical process. In GWAS, linear (for continuous traits, e.g. GFR or proteinuria) and logistic (for dichotomous traits, e.g. CKD or ESRD) regression models are used to calculate the mean shift in the distribution (for quantitative traits) or in the disease probability (for dichotomous phenotypes) per risk allele (compared to the other, the reference allele). By using the information on human genetic variation in the HAPMAP data set, these large data sets can be further enhanced by the imputation of a further over 1.5 million SNPs that lie between the genotyped SNPs [39]. In addition to the extension of the number of SNPs that can be studied, this makes the different SNP panels obtained from different genotyping platforms compatible across studies and is thus pivotal to pooling of several GWAS (GWAS metaanalyses, GWAMAs). The advantage of GWAMAs is that the larger sample size increases the power to detect small effects.

Since the genetic markers in genome-wide research are distributed across the whole genome, this analytical approach has the advantage of being unbiased by biological hypotheses, in contrast to candidate gene studies.

However, since a large number of statistical tests are performed, it is paramount to stringently correct for multiple testing. Applying the method described by Bonferroni to GWAS with 1 million independent SNP tests, an SNP association is deemed genome-wide significant if the $\mathrm{p}$ value is less than $5 \times 10^{-8}$ [40]. This small alpha level comes at the cost of a reduction in power. Very large sample sizes are thus required to identify a SNP association with genome-wide significance. Further, to confirm results of this first stage of locus discovery, confirmation in independent individuals is mandatory in a second stage of genetic testing of the SNPs identified in stage 1 GWAS [41].

A disadvantage of using current HAPMAP-imputed SNP data sets is that there is considerable uncertainty about the quality of information on SNPs with a minor allele frequency (MAF) below 10\% [42], which is further aggravated by the fact that most studies are underpow- 
ered to adequately analyze such SNPs. It is assumed that the low-frequency variants could account for larger effect sizes (fig. 1) [41]. The sequence information of several hundred individuals of European origin has become available with the 1000 Genomes data set in 2010 [42, 43], allowing the imputation of $>7$ million SNPs with improved coverage of such low-frequency variants. These data sets will be used in the near future and may close the gap between common variant approaches (GWAS) and rare mutation approaches (linkage analysis). The extent of the contribution of these low-frequency variants to the disease heritability is one of the current great debates with conclusions eagerly awaited.

\section{The Successes and Limitations of GWAS}

The first GWAS of common clinical diseases such as age-related macular degeneration [44], coronary heart disease [45-47] and diabetes mellitus types 1 and 2 [48] were published between 2005 and 2007, with successful studies of intermediate phenotypes, such as body mass index and blood glucose levels, as well as of traits without obvious pathological implications, such as eye and hair color, curly hair and freckling, following soon after [49].

By June 2010, a total of 904 published genome-wide associations with diseases and traits had been registered in the so-called GWAS catalog, hand-curated by the National Human Genetics Research Institute (USA) $[49,50]$. With some exceptions, common variants (MAF 10-50\%) with low effects are typically discovered: the increase in relative disease risk per copy of the risk allele is mostly $1-50 \%$, and the percent of variance explained (for quantitative traits) or the attributable disease risk (for dichotomous traits) usually falls short of 3\% (fig. 1) [41]. Only a handful of variants identified are associated with a more than 2-fold increased relative risk of disease, e.g. variants in the $C F H$ gene for age-related macular degeneration. The discrepancy between the mostly high levels of observed heritability of common diseases and traits (e.g. GFR has been shown to have a heritability of $36-75 \%$ ) and the small effect size attributable to the variants identified so far has been given the term 'missing heritability' [41, 51], with several explaining hypotheses:

(a) there are further frequent variants (MAF 10-50\%) with a smaller effect size, which GWAS with a larger sample size will detect, as has been the case e.g. for body mass index [52] and waist-hip ratio [53, 54];

(b) less frequent variants (MAF 1-10\%) account for larger effects and larger GWAS will detect them if their

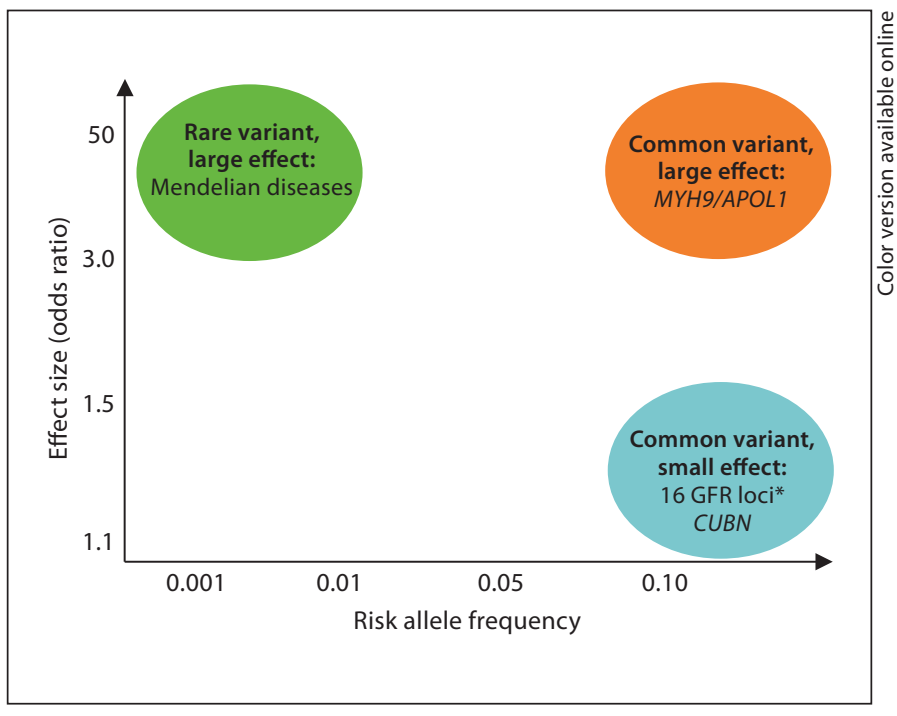

Fig. 1. Relation of effect size and risk allele frequency of SNPs associated with renal phenotypes. Adapted from McCarthy et al. [41]. * Published in Köttgen et al. [63, 79].

genotype data have a better coverage of these variants, which might be addressed to some extent by imputation to novel reference panels based on larger samples of sequenced individuals such as in the 1000 Genomes project [41-43];

(c) there is a great abundance of very rare variants $(\mathrm{MAF}<1 \%)$ with a large effect if taken jointly. If these are tagged by a common SNP, such a common SNP could be detected by GWAS. However, sequencing of specific loci in substantial sample sizes would be required to identify the rare variants;

(d) genetic variants other than SNPs comprise the functional entities, such as copy number variations or risk haplotypes. Some of these may be tagged by SNPs and could explain some of the found SNP associations [55]. Others might not be tagged by SNPs and will thus only be found by approaches alternative to SNP association;

(e) gene-gene and gene-environment interaction could dilute the main variant effect. Larger GWAS accounting for such interaction could detect SNPs involved in networks. However, the substantial sample sizes with detailed environmental phenotyping in sufficient quality are not yet available. The multitude of gene-gene interactions to be searched through would involve an enormous number of tests, increasing expo- 
nentially with increasing number of interacting SNPs considered. This is beyond the scope of currently available computing power.

Finally, an important caveat in the interpretation of all genetic association studies is that the SNPs identified through GWAS are rarely the causal variants, but rather implicate genetic loci for further functional study. Even the gene is usually not pinpointed by the GWAS top hit, as the SNP could tag any functional entity within the reach of any correlation of this SNP. So the first challenge after GWAS is pinpointing the gene involved, and the next is identifying the causal functional entity.

\section{Definition of Kidney Traits and Study Design}

CKD (here defined as GFR $<60 \mathrm{ml} / \mathrm{min} / 1.73 \mathrm{~m}^{2}$ ) has multiple etiologies, with the main causes in the general population with European descent being diabetic nephropathy and hypertensive nephropathy. Until the recent discovery of genetic variants with very strong effects on nondiabetic ESRD risk (see the section on GWAS of ESRD in African Americans) [56-58], this was also assumed for African Americans.

The involved mechanisms at CKD initiation may differ from those during kidney function decline through the progressive stages leading to ESRD as the most severe clinical endpoint [59-61], while certain histological changes during progression of $\mathrm{CKD}$, e.g. tubulointerstitial fibrosis, are common to multiple causes of CKD. Further, the genetic determinants may be distinct for the traits that define kidney disease, namely proteinuria and GFR, as previously proposed for disorders such as diabetic nephropathy [6]. Thus, trait definition and study design will invariably affect results of genetic analyses $[12,62]$.

It is unfeasible to measure GFR in large general population studies. Instead, GFR is estimated from serum creatinine, ideally complemented by GFR estimated from serum cystatin $\mathrm{C}$. The advantage of using two different biomarkers for estimation of GFR is that genetic factors affecting their production, metabolism and secretion can be evaluated [63].

Owing to the large sample size, cross-sectional analyses of estimated GFR (eGFR) and albuminuria as continuous traits have the highest power, while dichotomizing at established boundaries (e.g. eGFR: $60 \mathrm{ml} / \mathrm{min} /$ $1.73 \mathrm{~m}^{2}$; albumin-creatinine ratio: $\left.30 \mathrm{mg} / \mathrm{g}[64,65]\right)$ sacrifices power but provides a clinically relevant phenotype. The main drawback of cross-sectional studies is that the clinically highly relevant phenotypes kidney function decline, CKD initiation and progression of CKD cannot be studied. By calculating the annual decline in eGFR from data obtained longitudinally, factors affecting the slope of decline at all stages of CKD and the decline to CKD stage 3 can be investigated [62, 66-69]. The mechanisms involved in these phenotypes may differ in those without and with baseline CKD [59-61]: the former depicts persons that are still in the preclinical range of GFR and might drop towards CKD during the observation time, while the latter are already patients and mechanisms account for severe and/or rapid aggravation of the disease.

Longitudinal studies are thus suited to analyze CKD initiation, mechanisms involved in preclinical kidney function decline, and progression of CKD. However, especially in general population-based cohorts, studying mechanisms leading to ESRD is limited by low power owing to the small number of individuals progressing to ESRD. For example, in 17 years of follow-up in one of the largest cohorts (the ARIC study), only 101 (0.9\%) of 11,677 initially healthy individuals of European descent progressed to ESRD [70]. Thus, case-control studies of ESRD are the mainstay of the genetic study of this phenotype in spite of acknowledged methodological limitations of less than perfect comparability of cases and controls (possible confounding effects introduced by survival bias and differences across studies in access to renal replacement therapies between health care systems) $[41,71]$.

In principle, the above also applies to the much-studied phenotype of diabetic nephropathy, the main cause of ESRD in patients with European descent. In the subgroup of about $25 \%$ of all diabetes patients affected [72], this disorder progresses over many years of diabetes duration from normal albuminuria and GFR through mostly overlapping stages of hyperfiltration and increased GFR, mildly elevated albuminuria, declining GFR in the normal range, decrease of GFR to below $60 \mathrm{ml} / \mathrm{min} /$ $1.73 \mathrm{~m}^{2}$, overt and sometimes nephrotic-range proteinuria, decrease of GFR below $30 \mathrm{ml} / \mathrm{min} / 1.73 \mathrm{~m}^{2}$ and finally ESRD [73]. In early stages, regression to normalbuminuria is frequently observed [74]. Importantly, histological proof of this diagnosis is seldom obtained due to lack of clinical consequence.

Since kidney histology is typically not available, clinical criteria are used to reduce misclassification in casecontrol studies of diabetic nephropathy, though there is no uniform definition of diabetic nephropathy status across all genetic studies. Controls are mostly defined as 
those with long-standing diabetes and normal-range albuminuria ( $<30 \mathrm{mg}$ per day); the presence of a GFR $\geq 60$ $\mathrm{ml} / \mathrm{min} / 1.73 \mathrm{~m}^{2}$ is not always taken as a criterion defining controls [75]. Cases are mostly those with overt proteinuria (defined mostly as $>300 \mathrm{mg}$ per day) and/or ESRD in the absence of other possible causes of ESRD [76-78]. The presence of diabetic retinopathy is required by some studies $[15,75]$ since this makes the diagnosis of diabetic nephropathy more likely.

\section{Genetics of Kidney Traits}

\section{GWAS of GFR}

Recently, a first GWAMA on eGFR and CKD reported 3 novel susceptibility loci, i.e. UMOD, STC1 and SHROOM3 [79]. Soon thereafter, the CKDGen consortium, encompassing 67,093 individuals from 20 international, mostly general population-based cohort studies, identified 20 additional novel renal loci. Of these, 13 appear relevant for renal function, and 7 relevant for creatinine production, metabolism and secretion [63]. The genes located around or near the 16 renal function loci potentially influence calcium and phosphate homeostasis (STC1), the production of Tamm-Horsfall protein (UMOD), epithelial cell shape regulation (SHROOM3), nephrogenesis (ALMS1, VEGFA, potentially also DACH1), glomerular filtration barrier integrity and podocyte function (DAB2, VEGFA), angiogenesis (VEGFA), metabolic kidney function (PRKAG2, potentially GCKR, LASS2), ciliary function (ALMS1, GCKR-IFT172) and solute transport (SLC7A9, SLC34A1). The renal expression or role in the renal function of genes in 4 further loci (TFDP2, UBE2Q2, PIP5K1B, ATXN2/SH2B3) is unknown. Two of the 16 renal function loci were confirmed in simultaneously published work in independent cohorts (ALMS1 and SLC7A9) [80].

These 16 loci accounted for $1.4 \%$ of the variation in eGFR, and the effect size on risk for prevalent CKD did not exceed $20 \%$ relative risk (fig. 1). To assess the combined role of these 16 loci on renal function and CKD prevalence, a genetic risk score was computed that categorized the individuals by number of eGFR-lowering alleles per individual (possible range of risk score: 0-32). Across categories of the genetic risk score with a sample size of at least $\mathrm{n}=1,000$ per category, the mean eGFR dropped from $90.7 \mathrm{ml} / \mathrm{min} / 1.73 \mathrm{~m}^{2}$ (in individuals with a risk score of $11.5-12.5$ ) to $82.1 \mathrm{ml} / \mathrm{min} / 1.73 \mathrm{~m}^{2}$ (in individuals with a risk score of 22.5-23.5), and CKD prevalence ranged from 5.8 to $10.5 \%$, respectively (fig. 2).

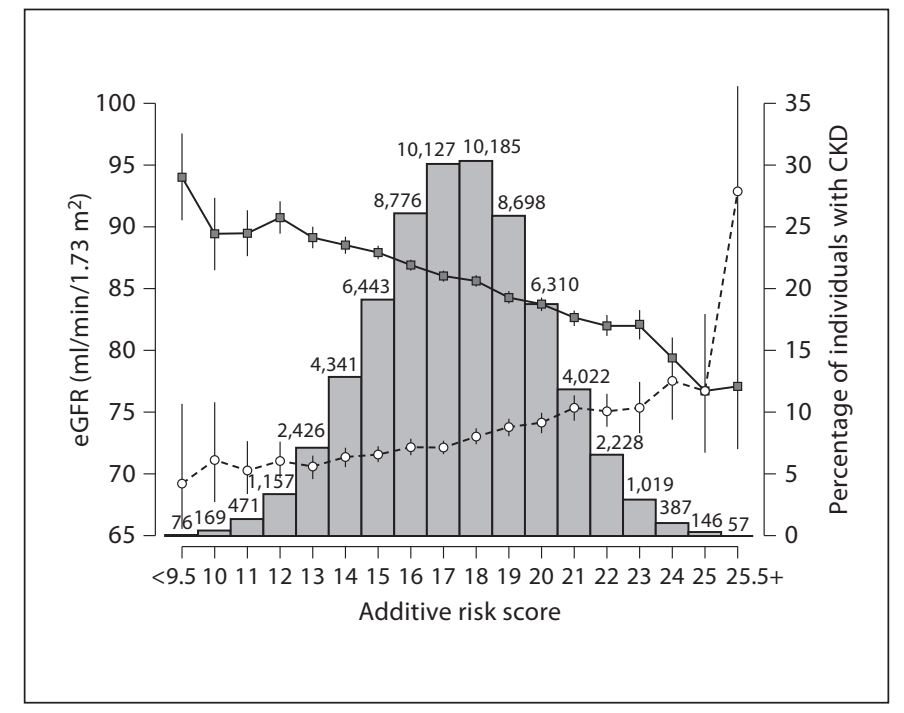

Fig. 2. Distribution of additive genetic risk score and eGFR (in ml/ $\mathrm{min} / 1.73 \mathrm{~m}^{2}$ ) and CKD prevalence (in \%) per risk score category. The additive risk score is calculated by summing the dosages of eGFR-lowering alleles of the 16 SNPs identified in Köttgen et al. [63] in CKDGen stage 1 meta-analysis $(n=67,093)$. Reproduced from Köttgen et al. [63] with permission of the Nature Publishing Group.

\section{GWAS of Albuminuria}

Albuminuria is another important parameter for kidney function and damage, with a urinary albumin-creatinine ratio $>30 \mathrm{mg} / \mathrm{g}$ being the first sign of kidney damage especially in diabetes mellitus. The CKDGen consortium conducted the first GWAMA of urinary albumin-creatinine ratio in 12 studies of the general population involving 31,580 participants and follow-up of selected loci in 15 additional studies including 27,746 participants. One nonsynonymous coding SNP in the CUBN gene was identified (MAF 10\%), explaining $0.2 \%$ of total variance of albuminuria (fig. 1). The results were validated in cohorts of African Americans and in a prospective cohort study of diabetes type 1 [81]. This gene locus is biologically plausible, since its gene product, cubilin, is expressed in the apical brush border of the kidney's proximal tubulus, and together with the proteins megalin and amnionless plays a key role in the receptor-mediated endocytosis of low-molecular-weight proteins; patients lacking cubilin or amnionless in autosomal recessive Imerslund-Gräsbeck disease (OMIM No. 261100) have variable levels of proteinuria due to inefficient proximal tubular protein reabsorption [82]. 
The intronic SNP in SHROOM3 associated with lower eGFR in the work by Köttgen et al. [63, 79] was the second highest-ranking SNP for lower levels of albuminuria in the GWAS of albuminuria, but genome-wide significance was not achieved [81]. No other loci associated with eGFR and CKD $[63,79]$ showed genome-wide significance in the GWAS of albuminuria or vice versa [81], supporting the proposed concept of disparate susceptibility genes for these renal function measures [6].

\section{GWAS of ESRD and Diabetic Nephropathy}

In 2003, Tanaka et al. [83] published the first GWAS of diabetic nephropathy in a Japanese population using a gene-based panel of over 55,000 SNPs in 94 cases of diabetic nephropathy and 94 controls, following the highestranking SNPs in a larger set of type 1 diabetes patients. SNPs in SLC12A3, the gene encoding the thiazide-sensitive sodium chloride cotransporter and mutated in Gitelman's syndrome, were associated with diabetic nephropathy. Some years later, an analysis in the same subjects with a total of over 80,000 gene-based SNPs revealed ELMO1 (engulfment and cell motility 1) and $A C A C B$ (acetyl coenzyme A carboxylase beta) as potential loci for diabetic nephropathy, validated by functional studies for both genes [75, 84].

ELMO1 was also among the loci associated with diabetic nephropathy in patients of European descent with type 1 diabetes in a genome-wide analysis in the GoKinD collection of patients, where cases are those with ESRD or those with overt proteinuria [85]. Further loci uncovered in this study were ZMIZ1, IRS2, TMPO, BID, KLRA1 and $C N D P 1$. The association with ELMO1 was confirmed in a candidate gene study in a larger sample from the GoKinD collective [86]. The highest-ranking SNPs were more significantly associated with diabetic nephropathy when those with proteinuria but without ESRD were excluded from analysis. Further validation in other ethnicities comes from studies that fine-mapped ELMO1 in a study of African Americans with type 2 diabetes-associated ESRD and nondiabetic controls [87], and in Pima Indians, though effect directions were not consistent [88]. Since the SNPs identified by all studies on diabetic nephropathy are not completely correlated, are not directionally consistent and did not reach genome-wide significance, further work is required to elucidate the mechanisms by which genetic variants in this locus affect diabetic nephropathy risk.

In a genome-wide analysis of over 100,000 SNPs in Pima Indians, Hanson et al. [89] identified plasmocytoma variant 1 (PVT1) as a susceptibility locus for
ESRD due to diabetes type 2, which was confirmed in Caucasians with ESRD attributed to diabetes type 1 [90].

Further, FRMD3 and CARS were associated with diabetic nephropathy in a large GWAS of patients with type 1 diabetes from the GoKinD collection [76]. Though none of the reported SNPs reached genome-wide significance, these findings were confirmed by an independent, prospective study of type 1 diabetes patients (DCCT/ EDIC [91]), and by expression in human kidney.

\section{GWAS of ESRD in African Americans}

After adjusting for socioeconomic factors, African Americans have an age-adjusted risk for ESRD that is almost 4-fold higher than that of European Americans [92]. In 2008, two groups simultaneously uncovered multiple common variants in MYH9 associated with an up to 7-fold risk for nondiabetic ESRD and focal segmental glomerulosclerosis, thus explaining most of the excess risk for these diseases in African Americans (fig. 1) $[56,57]$. Interestingly, 2 years later, it was shown that this risk is apparently conferred by SNPs in the neighboring gene, APOL1, that are in linkage disequilibrium with the originally described SNPs in MYH9. Intriguingly, the SNPs in APOL1 cause nonsynonymous amino acid exchanges, which appear to bear an evolutionary advantage by protecting from sleeping sickness due to Trypanosoma brucei rhodesiense [58]. Overall, these findings have incited a discussion that the majority of African Americans with nondiabetic ESRD may have a genetically determined ESRD disease entity, tentatively named MYH9/APOL1 nephropathy [93]. However, further work is required to unravel the mechanisms by which the variants in this locus lead to ESRD [94].

The search for genetic variants associated with ESRD due to diabetic nephropathy in African Americans has not been as successful [95]. A recent GWAS of ESRD in African Americans with type 2 diabetes found no SNPs with genome-wide significance, but several loci were named as potential candidates for the disease, with odds ratios similarly scaled as in GWAS for diabetic nephropathy in patients of European descent (range: odds ratio $0.57-1.54$ for the minor allele) [96]. The formally significant association of variants in MYH9 with diabetic nephropathy in a candidate gene study may be attributed to underlying nondiabetic kidney disease since the patients had not received kidney biopsy [97]. 


\section{IgA Nephropathy and Idiopathic Membranous}

Nephropathy

Recently, GWAS have been published for two immunological kidney disease entities: IgA nephropathy and idiopathic membranous nephropathy [98-100]. In both, genome-wide significant associations are found in a set of genetic loci, which confer a large effect size when analyzed jointly. The mechanisms by which the uncovered genetic variants cause the two diseases are unknown, but a role in modifying the immune response to allo- or autoantigens appears plausible. Interestingly, the second strong genetic signal for membranous nephropathy was in $P L A_{2} R 1$, the gene encoding the M-type phospholipase $\mathrm{A}_{2}$ receptor. This receptor is expressed in kidney podocytes and is one of the targets of autoantibodies leading to idiopathic membranous nephropathy [101-103]. The graded risk for membranous nephropathy with increasing numbers of risk alleles at both loci suggests that genetic modifications in the immune system in combination with genetic modifications in a podocyte gene leads to the production of autoantibodies, though experimental proof of this hypothesis is lacking. Further work is required also to identify the causal variants. Given the very low prevalence of this disease and the high risk allele frequency of variants detected in this publication (risk allele frequency at rs2187668 in HLA-DQA1: 39.2\%; risk allele frequency at rs4664308 in $P L A_{2} R 1: 25.2 \%$ ), it can be expected that the detected variants are in linkage disequilibrium with multiple rare causative variants.

\section{Kidney Function Decline, CKD Initiation and \\ Progression of CKD}

The mechanisms underlying CKD initiation and progression of CKD to ESRD may differ [59-61, 104], and understanding the genetics of these traits could lead to the development of novel diagnostic and therapeutic tools. However, GWAS examining these traits have not been performed, while candidate gene studies have recently been published $[105,106]$.

\section{Conclusion}

In conclusion, major advances have been made in the past decade by GWAS to unravel the genetics of complex diseases including kidney diseases. While loci unequivocally associated with risk for diabetic nephropathy remain to be discovered, several novel loci associated with kidney function, prevalent CKD, albuminuria and certain immunological kidney diseases have been uncovered in individuals of European descent. Common variants in the MYH9/APOL1 locus account for most of the excess risk for nondiabetic ESRD in African Americans when compared to their counterparts of European descent, but the mechanisms involved are yet unknown. Finally, further work is required to discover genetic variants associated with change in renal function over time, and genetic marker panels with higher resolution will enable the analysis of less frequent variants on a genomewide scale targeted to close the gap between the estimated heritability and the current genetically explained disease risk. The understanding of the genetic underpinning of several aspects of kidney diseases will greatly foster grasping the involved pathways with the hope for improved diagnostic and therapeutic tools in the future.

\section{Disclosure Statement}

The authors declare that they have nothing to disclose.

\section{References}

$\checkmark 1$ Baumeister SE, Böger CA, Krämer BK, et al: Effect of chronic kidney disease and comorbid conditions on health care costs: a 10-year observational study in a general population. Am J Nephrol 2010;31:222-229.

-2 Levey AS, Atkins R, Coresh J, et al: Chronic kidney disease as a global public health problem: approaches and initiatives - a position statement from Kidney Disease Improving Global Outcomes. Kidney Int 2007;72:247259.
- 3 Matsushita K, van der Velde M, Astor BC, et al: Association of estimated glomerular filtration rate and albuminuria with all-cause and cardiovascular mortality in general population cohorts: a collaborative meta-analysis. Lancet 2010;375:2073-2081.

-4 Fox CS, Larson MG, Leip EP, Culleton B, Wilson PW, Levy D: Predictors of new-onset kidney disease in a community-based population. JAMA 2004;291:844-850.

5 O'Seaghdha CM, Fox CS: Genetics of chronic kidney disease. Nephron Clin Pract 2011; 118:c55-c63.
6 Placha G, Canani LH, Warram JH, Krolewski AS: Evidence for different susceptibility genes for proteinuria and ESRD in type 2 diabetes. Adv Chronic Kidney Dis 2005;12:155-169.

7 Hildebrandt F: Genetic kidney diseases. Lancet 2010;375:1287-1295.

8 Patrakka J, Tryggvason K: New insights into the role of podocytes in proteinuria. Nat Rev Nephrol 2009;5:463-468.

-9 Tryggvason K, Patrakka J, Wartiovaara J: Hereditary proteinuria syndromes and mechanisms of proteinuria. N Engl J Med 2006;354:1387-1401. 
10 Hinkes BG, Mucha B, Vlangos CN, et al: Nephrotic syndrome in the first year of life: two thirds of cases are caused by mutations in 4 genes (NPHS1, NPHS2, WT1, and LAMB2). Pediatrics 2007;119:e907-e919.

-11 McKnight AJ, Currie D, Maxwell AP: Unravelling the genetic basis of renal diseases; from single gene to multifactorial disorders. J Pathol 2010;220:198-216.

- 12 Estrella MM, Sperati CJ, Kao WH, Parekh RS: Genetic epidemiology of chronic kidney disease. Curr Opin Nephrol Hypertens 2010; 19:283-291.

$\checkmark 13$ Köttgen A: Genome-wide association studies in nephrology research. Am J Kidney Dis 2010;56:743-758.

14 Janssen B, Hohenadel D, Brinkkoetter P, et al: Carnosine as a protective factor in diabetic nephropathy: association with a leucine repeat of the carnosinase gene CNDP1. Diabetes 2005;54:2320-2327.

15 Vardarli I, Baier LJ, Hanson RL, et al: Gene for susceptibility to diabetic nephropathy in type 2 diabetes maps to 18q22.3-23. Kidney Int 2002;62:2176-2183.

-16 Bowden DW, Colicigno CJ, Langefeld CD, et al: A genome scan for diabetic nephropathy in African Americans. Kidney Int 2004;66 1517-1526.

17 Freedman BI, Hicks PJ, Sale MM, et al: A leucine repeat in the carnosinase gene CNDP1 is associated with diabetic end-stage renal disease in European Americans. Nephrol Dial Transplant 2007;22:1131-1135.

-18 Iyengar SK, Abboud HE, Goddard KA, et al: Genome-wide scans for diabetic nephropathy and albuminuria in multiethnic populations: the family investigation of nephropathy and diabetes (FIND). Diabetes 2007;56: 1577-1585.

$\checkmark 19$ McDonough CW, Hicks PJ, Lu L, Langefeld CD, Freedman BI, Bowden DW: The influence of carnosinase gene polymorphisms on diabetic nephropathy risk in African-Americans. Hum Genet 2009;126:265-275.

20 Fox CS, Yang Q, Cupples LA, et al: Genomewide linkage analysis to serum creatinine, GFR, and creatinine clearance in a community-based population: the Framingham Heart Study. J Am Soc Nephrol 2004; 15: 2457-2461.

-21 Fox CS, Yang Q, Guo CY, et al: Genome-wide linkage analysis to urinary microalbuminuria in a community-based sample: the Framingham Heart Study. Kidney Int 2005; 67:70-74.

22 Hunt SC, Coon H, Hasstedt SJ, et al: Linkage of serum creatinine and glomerular filtration rate to chromosome 2 in Utah pedigrees. Am J Hypertens 2004;17:511-515.

23 Hunt SC, Hasstedt SJ, Coon H, et al: Linkage of creatinine clearance to chromosome 10 in Utah pedigrees replicates a locus for endstage renal disease in humans and renal failure in the fawn-hooded rat. Kidney Int 2002; 62:1143-1148.
24 DeWan AT, Arnett DK, Atwood LD, et al: A genome scan for renal function among hypertensives: the HyperGEN study. Am J Hum Genet 2001;68:136-144.

25 Freedman BI, Beck SR, Rich SS, et al: A genome-wide scan for urinary albumin excretion in hypertensive families. Hypertension 2003;42:291-296.

$26 \mathrm{He}$ B, Osterholm AM, Hoverfalt A, et al: Association of genetic variants at $3 \mathrm{q} 22$ with nephropathy in patients with type 1 diabetes mellitus. Am J Hum Genet 2009;84:5-13.

27 Imperatore G, Hanson RL, Pettitt DJ, Kobes S, Bennett PH, Knowler WC: Sib-pair linkage analysis for susceptibility genes for microvascular complications among Pima Indians with type 2 diabetes. Pima Diabetes Genes Group. Diabetes 1998;47:821830.

28 Krolewski AS, Poznik GD, Placha G, et al: A genome-wide linkage scan for genes controlling variation in urinary albumin excretion in type II diabetes. Kidney Int 2006;69:129136.

29 Placha G, Poznik GD, Dunn J, et al: A genome-wide linkage scan for genes controlling variation in renal function estimated by serum cystatin $C$ levels in extended families with type 2 diabetes. Diabetes 2006;55:3358 3365 .

30 Rogus JJ, Poznik GD, Pezzolesi MG, et al: High-density single nucleotide polymorphism genome-wide linkage scan for susceptibility genes for diabetic nephropathy in type 1 diabetes: discordant sibpair approach. Diabetes 2008;57:2519-2526.

31 Turner ST, Kardia SL, Mosley TH, Rule AD, Boerwinkle E, de Andrade M: Influence of genomic loci on measures of chronic kidney disease in hypertensive sibships. J Am Soc Nephrol 2006;17:2048-2055.

32 Osterholm AM, He B, Pitkaniemi J, et al: Genome-wide scan for type 1 diabetic nephropathy in the Finnish population reveals suggestive linkage to a single locus on chromosome 3q. Kidney Int 2007;71:140-145.

33 Schelling JR, Abboud HE, Nicholas SB, et al: Genome-wide scan for estimated glomerular filtration rate in multi-ethnic diabetic populations: the Family Investigation of $\mathrm{Ne}$ phropathy and Diabetes (FIND). Diabetes 2008; $57: 235-243$.

34 Maeda S: Genetics of diabetic nephropathy. Ther Adv Cardiovasc Dis 2008;2:363-271.

- 35 Hirschhorn JN, Lohmueller K, Byrne E, Hirschhorn K: A comprehensive review of genetic association studies. Genet Med 2002; 4:45-61.

36 Lander ES, Linton LM, Birren B, et al: Initial sequencing and analysis of the human genome. Nature 2001;409:860-921.

37 Venter JC, Adams MD, Myers EW, et al: The sequence of the human genome. Science 2001;291:1304-1351.

38 International HapMap Consortium: A haplotype map of the human genome. Nature 2005;437:1299-320.
39 Marchini J, Howie B, Myers S, McVean G, Donnelly P: A new multipoint method for genome-wide association studies by imputation of genotypes. Nat Genet 2007;39:906913.

40 Pe'er I, Yelensky R, Altshuler D, Daly MJ: Estimation of the multiple testing burden for genomewide association studies of nearly all common variants. Genet Epidemiol 2008;32: 381-385.

41 McCarthy MI, Abecasis GR, Cardon LR, et al: Genome-wide association studies for complex traits: consensus, uncertainty and challenges. Nat Rev Genet 2008;9:356-369.

42 Durbin RM, Abecasis GR, Altshuler DL, et al: A map of human genome variation from population-scale sequencing. Nature 2010; 467:1061-1073.

43 The 1000 Genomes Project. http://www. 1000genomes.org.

44 Klein RJ, Zeiss C, Chew EY, et al: Complement factor $\mathrm{H}$ polymorphism in age-related macular degeneration. Science 2005;308: 385-389.

45 McPherson R, Pertsemlidis A, Kavaslar N, et al: A common allele on chromosome 9 associated with coronary heart disease. Science 2007;316:1488-1491.

-46 Helgadottir A, Thorleifsson G, Manolescu A et al: A common variant on chromosome 9p21 affects the risk of myocardial infarction. Science 2007;316:1491-1493.

-47 Samani NJ, Erdmann J, Hall AS, et al: Genomewide association analysis of coronary artery disease. N Engl J Med 2007;357:443453.

48 Wellcome Trust Case Control Consortium: Genome-wide association study of 14,000 cases of seven common diseases and 3,000 shared controls. Nature 2007;447:661-678.

49 Hindorff LA JH, Hall PN, Mehta JP, Manolio TA: A catalog of published genomewide association studies. www.genome. gov/gwastudies (accessed February 12, 2011).

50 Hindorff LA, Sethupathy P, Junkins HA, et al: Potential etiologic and functional implications of genome-wide association loci for human diseases and traits. Proc Natl Acad Sci USA 2009;106:9362-9367.

51 Manolio TA, Collins FS, Cox NJ, et al: Finding the missing heritability of complex diseases. Nature 2009;461:747-753.

52 Speliotes EK, Willer CJ, Berndt SI, et al: Association analyses of 249,796 individuals reveal 18 new loci associated with body mass index. Nat Genet 2010;42:937-948.

53 Lindgren CM, Heid IM, Randall JC, et al: Genome-wide association scan meta-analysis identifies three loci influencing adiposity and fat distribution. PLoS Genet 2009; 5:e1000508.

54 Heid IM, Jackson AU, Randall JC, et al: Meta-analysis identifies 13 new loci associated with waist-hip ratio and reveals sexual dimorphism in the genetic basis of fat distribution. Nat Genet 2010;42:949-960. 
-55 Willer CJ, Speliotes EK, Loos RJ, et al: Six new loci associated with body mass index highlight a neuronal influence on body weight regulation. Nat Genet 2009;41:25-34.

56 Kao WH, Klag MJ, Meoni LA, et al: MYH9 is associated with nondiabetic end-stage renal disease in African Americans. Nat Genet 2008;40:1185-1192.

- 57 Kopp JB, Smith MW, Nelson GW, et al: MYH9 is a major-effect risk gene for focal segmental glomerulosclerosis. Nat Genet 2008;40:1175-1184.

58 Genovese G, Friedman DJ, Ross MD, et al: Association of trypanolytic ApoL1 variants with kidney disease in African Americans. Science 2010;329:841-845.

59 Pillebout E, Burtin M, Yuan HT, et al: Proliferation and remodeling of the peritubular microcirculation after nephron reduction: association with the progression of renal lesions. Am J Pathol 2001;159:547-560.

-60 Schmid H, Boucherot A, Yasuda Y, et al: Modular activation of nuclear factor-kappaB transcriptional programs in human diabetic nephropathy. Diabetes 2006;55:2993-3003.

61 Viau A, El Karoui K, Laouari D, et al: Lipocalin 2 is essential for chronic kidney disease progression in mice and humans. J Clin Invest 2010;120:4065-4076.

62 Bash LD, Coresh J, Kottgen A, et al: Defining incident chronic kidney disease in the research setting: the ARIC Study. Am J Epidemiol 2009;170:414-424.

-63 Köttgen A, Pattaro C, Böger CA, et al: New loci associated with kidney function and chronic kidney disease. Nat Genet 2010;42: 376-384.

64 National Kidney Foundation: K/DOQI clinical practice guidelines for chronic kidney disease: evaluation, classification, and stratification. Am J Kidney Dis 2002;39:S1-S266.

-65 Levey AS, Eckardt KU, Tsukamoto Y, et al: Definition and classification of chronic kidney disease: a position statement from Kidney Disease: Improving Global Outcomes (KDIGO). Kidney Int 2005;67:2089-2100.

-66 Lindeman RD, Tobin J, Shock NW: Longitudinal studies on the rate of decline in renal function with age. J Am Geriatr Soc 1985;33: 278-285.

67 Rifkin DE, Shlipak MG, Katz R, et al: Rapid kidney function decline and mortality risk in older adults. Arch Intern Med 2008; 168: 2212-2218.

68 Shlipak MG, Katz R, Kestenbaum B, et al: Rate of kidney function decline in older adults: a comparison using creatinine and cystatin C. Am J Nephrol 2009;30:171-178.

-69 Shlipak MG, Katz R, Kestenbaum B, et al: Rapid decline of kidney function increases cardiovascular risk in the elderly. J Am Soc Nephrol 2009;20:2625-2630.

-70 Bash LD, Astor BC, Coresh J: Risk of incident ESRD: a comprehensive look at cardiovascular risk factors and 17 years of follow-up in the Atherosclerosis Risk in Communities (ARIC) Study. Am J Kidney Dis 2010;55:31-41.
Hallan SI, Coresh J, Astor BC, et al: International comparison of the relationship of chronic kidney disease prevalence and ESRD risk. J Am Soc Nephrol 2006; 17:2275-2284.

72 Adler AI, Stevens RJ, Manley SE, Bilous RW, Cull CA, Holman RR: Development and progression of nephropathy in type 2 diabetes: the United Kingdom Prospective Diabetes Study (UKPDS 64). Kidney Int 2003;63:225232.

73 Remuzzi G, Benigni A, Remuzzi A: Mechanisms of progression and regression of renal lesions of chronic nephropathies and diabetes. J Clin Invest 2006;116:288-296.

74 Steinke JM, Sinaiko AR, Kramer MS, Suissa $S$, Chavers BM, Mauer M: The early natural history of nephropathy in type 1 diabetes. 3 . Predictors of 5-year urinary albumin excretion rate patterns in initially normoalbuminuric patients. Diabetes 2005;54:21642171.

75 Shimazaki A, Kawamura Y, Kanazawa A, et al: Genetic variations in the gene encoding ELMO1 are associated with susceptibility to diabetic nephropathy. Diabetes 2005;54: 1171-1178.

76 Pezzolesi MG, Poznik GD, Mychaleckyj JC, et al: Genome-wide association scan for diabetic nephropathy susceptibility genes in type 1 diabetes. Diabetes 2009;58:1403-1410.

77 Böger CA, Haak T, Götz AK, et al: Effect of ACE and AT-2 inhibitors on mortality and progression to microalbuminuria in a nested case-control study of diabetic nephropathy in diabetes mellitus type 2: results from the GENDIAN study. Int J Clin Pharmacol Ther 2006;44:364-374.

78 Böger CA, Stubanus M, Haak T, et al: Effect of MTHFR C677T genotype on survival in type 2 diabetes patients with end-stage diabetic nephropathy. Nephrol Dial Transplant 2007;22:154-162.

79 Köttgen A, Glazer NL, Dehghan A, et al: Multiple loci associated with indices of renal function and chronic kidney disease. Nat Genet 2009;41:712-717.

80 Chambers JC, Zhang W, Lord GM, et al: Genetic loci influencing kidney function and chronic kidney disease. Nat Genet 2010;42: 373-375.

81 Böger C, Chen MH, Tin A, Olden M, Köttgen A, de Boer IH, et al: CUBN is a gene locus for albuminuria. J Am Soc Nephrol 2011;22: 555-570.

82 Christensen EI, Verroust PJ, Nielsen R: Receptor-mediated endocytosis in renal proximal tubule. Pflugers Arch 2009;458:10391048

83 Tanaka N, Babazono T, Saito S, et al: Association of solute carrier family 12 (sodium/ chloride) member 3 with diabetic nephropathy, identified by genome-wide analyses of single nucleotide polymorphisms. Diabetes 2003;52:2848-2853.
84 Maeda S, Kobayashi MA, Araki S, et al: A single nucleotide polymorphism within the acetyl-coenzyme A carboxylase beta gene is associated with proteinuria in patients with type 2 diabetes. PLoS Genet 2010;6: e1000842.

85 Craig DW, Millis MP, DiStefano JK: Genome-wide SNP genotyping study using pooled DNA to identify candidate markers mediating susceptibility to end-stage renal disease attributed to type 1 diabetes. Diabet Med 2009;26:1090-1098.

86 Pezzolesi MG, Katavetin P, Kure M, et al: Confirmation of genetic associations at ELMO1 in the GoKinD collection supports its role as a susceptibility gene in diabetic nephropathy. Diabetes 2009;58:2698-2702.

87 Leak TS, Perlegas PS, Smith SG, et al: Variants in intron 13 of the ELMO1 gene are associated with diabetic nephropathy in African Americans. Ann Hum Genet 2009;73: 152-159.

88 Hanson RL, Millis MP, Young NJ, et al: ELMO1 variants and susceptibility to diabetic nephropathy in American Indians. Mol Genet Metab 2010;101:383-390.

89 Hanson RL, Craig DW, Millis MP, et al: Identification of PVT1 as a candidate gene for end-stage renal disease in type 2 diabetes using a pooling-based genome-wide single nucleotide polymorphism association study. Diabetes 2007;56:975-983.

$\checkmark 90$ Millis MP, Bowen D, Kingsley C, Watanabe RM, Wolford JK: Variants in the plasmacytoma variant translocation gene (PVT1) are associated with end-stage renal disease attributed to type 1 diabetes. Diabetes 2007;56: 3027-3032.

91 Sustained effect of intensive treatment of type 1 diabetes mellitus on development and progression of diabetic nephropathy: the Epidemiology of Diabetes Interventions and Complications (EDIC) study. JAMA 2003; 290:2159-2167.

92 National Institute of Diabetes and Digestive and Kidney Disease (ed): US Renal Data System 2007 Annual Data Report: Atlas of Chronic Kidney Disease and End-Stage Renal Disease in the United States. Bethesda, National Institute of Diabetes and Digestive and Kidney Disease, 2007.

93 Freedman BI, Sedor JR: Hypertension-associated kidney disease: perhaps no more. J Am Soc Nephrol 2008;19:2047-2051.

94 Kronenberg F: APOL1 variants and kidney disease. There is no such thing as a free lunch. Nephrol Dial Transplant 2011;26: 775-778.

95 Bostrom MA, Freedman BI: The spectrum of MYH9-associated nephropathy. Clin J Am Soc Nephrol 2010;5:1107-1113.

96 McDonough CW, Palmer ND, Hicks PJ, et al: A genome-wide association study for diabetic nephropathy genes in African Americans. Kidney Int 2011;79:563-572. 
-97 Freedman BI, Hicks PJ, Bostrom MA, et al: Non-muscle myosin heavy chain 9 gene MYH9 associations in African Americans with clinically diagnosed type 2 diabetes mellitus-associated ESRD. Nephrol Dial Transplant 2009;24:3366-3371.

98 Feehally J, Farrall M, Boland A, et al: HLA has strongest association with IgA nephropathy in genome-wide analysis. J Am Soc Nephrol 2010;21:1791-1797.

99 Gharavi AG, Kiryluk K, Choi M, et al: Genome-wide association study identifies susceptibility loci for IgA nephropathy. Nat Genet 2011;43:321-327.
100 Stanescu HC, Arcos-Burgos M, Medlar A, et al: Risk HLA-DQA1 and PLA(2)R1 alleles in idiopathic membranous nephropathy. N Engl J Med 2011;364:616-626.

101 Beck LH, Jr., Bonegio RG, Lambeau G, et al: M-type phospholipase A2 receptor as target antigen in idiopathic membranous nephropathy. N Engl J Med 2009;361:11-21.

102 Debiec H, Guigonis V, Mougenot B, et al: Antenatal membranous glomerulonephritis due to anti-neutral endopeptidase antibodies. N Engl J Med 2002;346:2053-2060.

103 Prunotto M, Carnevali ML, Candiano G, et al: Autoimmunity in membranous nephropathy targets aldose reductase and SOD2. J Am Soc Nephrol 2010;21:507-519.
104 Kronenberg F: Emerging risk factors and markers of chronic kidney disease progression. Nat Rev Nephrol 2009;5:677-689.

105 Köttgen A, Hwang SJ, Rampersaud E, et al: TCF7L2 variants associate with CKD progression and renal function in populationbased cohorts. J Am Soc Nephrol 2008;19: 1989-1999.

106 Liu M, Shi S, Senthilnathan S, et al: Genetic variation of DKK3 may modify renal disease severity in ADPKD. J Am Soc Nephrol 2010;21:1510-1520. 